Published in final edited form as:

Nutr Clin Pract. 2019 June ; 34(3): 421-427. doi:10.1002/ncp.10194.

\title{
Body Composition with Dual-Energy X-ray Absorptiometry and Bio-electrical Impedance Analysis in Breast Cancer Survivors
}

\author{
Kyuwan Lee, MS, Nathalie Sami, BS, Frank Sweeney, BS, and Christina M. Dieli-Conwright, \\ PhD \\ Division of Biokinesiology and Physical Therapy, University of Southern California, Los Angeles, \\ CA, United States
}

\begin{abstract}
Objective: As obesity is recognized an independent risk factor for breast cancer recurrence, the appropriate assessment of body composition is crucial to guide weight management in breast cancer survivors (BCS). The purpose of this study was to assess whether Dual-Energy X-ray Absorptiometry (DXA) and Bio-electrical Impedance Analysis (BIA) yield similar results for body composition in BCS. We examined whether these results differed by BMI amongst our cohort.
\end{abstract}

\begin{abstract}
Methods: BCS (Stage I-III) who had completed cancer treatment within previous 6 months were included. Body fat percentage, lean body mass, and fat mass were estimated using DXA (GE Lunar Prodigy) and BIA (InBody 520) under fasting conditions in 89 BCS. BMI categories included: normal (18-24.99 kg/m²; $\mathrm{n}=28)$, overweight $\left(25.0-29.9 \mathrm{~kg} / \mathrm{m}^{2} ; \mathrm{n}=22\right)$, obese $\left(>30 \mathrm{~kg} / \mathrm{m}^{2} ; \mathrm{n}=23\right)$, and severely obese BCS $\left(>35 \mathrm{~kg} / \mathrm{m}^{2} ; \mathrm{n}=17\right)$. Agreement between the devices was assessed by Bland-Altman analysis.
\end{abstract}

Results: There was no agreement between the two devices for body fat percentage (DXA: $44.2 \pm 6.2 \%$ vs BIA: $40.4 \pm 7.8 \%$ ), lean body mass (DXA: $39.1 \pm 7.6 \mathrm{~kg}$ vs BIA: $42.9 \pm 5.9 \mathrm{~kg}$ ) and fat mass (DXA: $32.4 \pm 10.8 \mathrm{~kg}$ vs BIA: $30.6 \pm 11.0 \mathrm{~kg}$; $\mathrm{P}<0.001$ ). These findings were consistent in normal, overweight, and obese BCS. There was agreement between the two devices for fat mass (DXA: $48.7 \pm 7.2 \mathrm{~kg}$ vs BIA: $47.9 \pm 5.7 \mathrm{~kg}$ ) in severely obese BCS ( $\mathrm{P}=0.102)$, possibly due to small sample size.

Conclusions: BIA may underestimate body fat percentage and fat mass and overestimate lean body mass, compared to DXA. Future studies are warranted to assess the utilization of these two devices in a larger cohort of BCS within BMI categories.

\section{Keywords}

absorptiometry; body composition; breast cancer; electric impedance; neoplasms

\footnotetext{
Contact information: Christina M. Dieli-Conwright, Division of Biokinesiology and Physical Therapy University of Southern California, 1540 E. Alcazar St., CHP 155 Los Angeles, CA 90089, cdieli@usc.edu.

Disclosure: The authors declared no conflict of interest

Conflict of Interest Statement: None declared.

Clinical Trial Registration: NCT01140282
} 


\section{Introduction}

As obesity is increasingly recognized as a risk factor for breast cancer and its recurrence, the assessment of body composition becomes vital to understanding overall health in breast cancer survivors (BCS).[1] Although the association between body mass index (BMI) and breast cancer risk and recurrence has been extensively studied among breast cancer survivors, body composition (i.e. fat and lean mass) is recognized as an indicator of poor prognosis which stimulates cancer progression through chronic inflammation in white adipose tissue in breast cancer survivors. [2] BCS have a two- to three-fold higher prevalence of obesity than other cancer survivors (i.e. kidney, pancreas, and thyroid cancer), and it is estimated that obesity will cause more than 500,000 additional cases of cancer in the U.S. by 2030.[3] Body composition in BCS has been measured by a variety of methods including dual-energy X-ray absorptiometry (DXA),[4] computed tomography,[5] bioelectrical impedance analysis (BIA),[6] skinfolds,[7] and magnetic resonance imaging (MRI)[8] to describe and lower excessive body components (i.e., central adiposity, body fat mass) known to be related to increased risk of cancer mortality and recurrence in BCS. Each procedure has distinct advantages and disadvantages (i.e. accuracy, feasibility, cost, and availability), however, some procedures may be limited for research investigations with BCS due to availability and cost, [9] and may contribute to unreliable results of said clinical studies.

DXA is a reliable measure for assessing body composition as it requires little effort of the patient and has sound psychometric properties including high validity and reproducibility. $[10,11]$ However, the use of DXA is limited due to availability and high cost. In contrast, BIA is a more commonly used tool to estimate body composition due to safety (no x-ray involved), accuracy, low cost and ease of use.[12,13] Clinical breast studies have extensively utilized BIA as a component of the clinical exam, however, little is known about body composition values calculated by BIA in BCS, and how it is related to those same values assessed by DXA. Particularly, BIA measurements are mostly influenced by a fasting or a fed state. Temporary exercise can affect the accuracy of measurements by 3-8\% decrease, and thus BIA measurement must be standardized with caution. Despite these limitations, BIA is non-invasive, low variations and can be performed in any clinical populations

BIA may be a practical alternative measure for DXA when participants are classified by BMI categories (i.e. normal, overweight, and obese).[14] Volgyi et al. (2008) evaluated to what extent DXA and two different types of BIA yield similar results for body fat mass in 82 men and 86 women across different levels of obesity. BIA showed on average 2-6\% lower value for fat mass in normal BMI men and women in all BMI categories. The difference between BIA and DXA was smaller in overweight and obese women when measuring body fat percentage, which implies feasible use of BIA in overweight and obese BCS. This study, however, is limited in that body fat mass remains significantly different in BIA versus DXA measurements based on their results; body fat percentage is the only measure that is not significantly different per BIA and DXA. Consequently, this inconsistent data may influence the use of body composition assessments in BCS in the clinical oncology setting. With such 
broad use of BIA in these settings currently, investigation is warranted into examining the impact of BMI on accurately assessing body composition in BCS.

The purpose of this study was two-fold: 1) to assess whether DXA and BIA yield similar results for body composition including fat mass, body fat percentage and lean mass in BCS, and 2) to assess whether the body composition results differed between the 2 devices within BMI categories in BCS. We hypothesized: 1) BIA and DXA would have similar estimations of body composition: fat mass, body fat percentage, and lean body mass, and 2) body composition would not differ between DXA and BIA based on BMI in BCS.

\section{Methods}

\section{Study Design}

All assessments were performed as part of a larger ongoing clinical trial designed to examine the effects of aerobic and resistance exercise on metabolic syndrome in overweight and obese BCS.[15] Participants visited the lab as part of their baseline visit and body composition assessments were performed under fasting conditions and similar testing order. A licensed DXA technician performed the assessments for all participants. In brief, the assessments were performed between 7:00-11:00 AM, in a temperature-controlled room, after a 10-hour fast, and abstinence from alcohol, caffeine, and vitamins. With the exception of food, alcohol, caffeine, and vitamins, participants were instructed to drink water if needed. After 10 minutes of quiet rest, participants underwent BIA assessment using the InBody 520 Body Composition Analyzer (BioSpace, Cerritos, CA, USA) in a normal standing position with arms stretched at 15 degrees off the body during the analysis. Once BIA assessment was completed, participants underwent a whole body DXA scan. All scans were performed by the same licensed DXA technician using the same equipment and processed according to the manufacturer's instructions for a whole-body scan.

\section{Study Population}

Participants were included based on the following inclusion criteria: 1) newly diagnosed (IIII) with a first primary invasive breast cancer, 2) over the age of 18 years, 3) had undergone a lumpectomy or mastectomy, 4) completed neoadjuvant/adjuvant chemotherapy and/or radiation therapy within the previous 6 months, 5) BMI $>25 \mathrm{~kg} / \mathrm{m}^{2}$ and/or body fat $>30 \%, 6$ ) Participated in less than 60 minutes of physical activity per week at time of study enrollment, 7) Nonsmokers, 8) Willing to travel to the exercise facility, and 8) Able to provide physician clearance to participate in exercise program. Exclusion criteria included 1) diabetes, uncontrolled hypertension or thyroid disease, 2) weight reduction $\geq 10 \%$ within past 6 months, 3) metastatic disease, 4) planned reconstructive surgery, 5) pulmonary disease, angina, atrial fibrillation, myocardial infarction, and stroke. The study was reviewed and approved by the Institutional Review Board.

\section{Anthropometrics.}

Height and weight were measured on a medical scale (Detecto® 437, Webb City, MO) and used to calculate BMI by the same experienced tester for all participants. 


\section{BIA assessment.}

The InBody 520 Body Composition Analyzer (BioSpace, Cerritos, CA, USA) was used to measure body composition using eight points of tactile electrodes (i.e. two thumb, two palm, two front sole, and two rear sole electrodes). Participants were asked not to perform any physical tasks and were restrained from eating 4 hours before testing according to the manufacturer's instruction but participants were allowed to drink water. Age, height, and sex were entered on the touch screen before the device starts measuring body composition. Participants stood on the device with bare feet in contact with the electrode foot pads. Body weight was measured by its own system built in the device. The device detects intracellular and extracellular water levels separately using multi-frequency impedance plethysmograph body composition analyzer, which measures reactance at three specific frequencies $(5 \mathrm{kHz}$, $50 \mathrm{kHz}$, and $250 \mathrm{kHz}$ ) to produce a total of 15 impedance values for 5 body segments [16]. Outcomes obtained from the device included lean body mass, fat mass, and body fat percentage.

\section{DXA assessment.}

DXA (DPX-IQ 2288 with Smart Scan version 4.7e; Lunar Radiation Corporation, Madison, WI, USA) was used to measure fat mass and body fat percentage and LM using a wholebody scan. Full-quality assurance procedures were carried out daily using a bio-imaging phantom acrylic block (VCP-057; Bio-Imaging Technologies, Inc., Newtown, PA, USA) according to the instructions for use, which included a calibration of functionality, accuracy, andprecision of densitometer. Participants were asked to wear comfortable exercise clothing and remove items that can attenuate the x-ray beam including jewelry, body piercings, hair accessories, and clothing with zippers, snaps, buckles, and buttons. Participants were positioned supine on the bed of the DXA scanner, with arms slightly abducted. A total body scan began at the subject's head and scanned the entire body until completion at the feet. Each scan lasted less than 10 minutes using a low-dose radiation x-ray and was performed by a California state licensed DXA technician.

\section{Statistical analysis.}

Data were checked for normality by Shapiro-Wilk's W test prior to analysis. Descriptive results are reported as means \pm SD. A $P$ value of $<0.05$ was considered statistically significant. The Bland-Altman method was used to determine measures of agreement between body composition from DXA and BIA. To assess differences in the 2 devices based on body size, participants were categorized into 4 groups by BMI; normal, overweight, obese, and severely obese BCS. BMI groups were defined according to national standards (National Institutes of Health), normal BMI 18-24.99 kg/m²; overweight BMI 25.0-29.9 $\mathrm{kg} / \mathrm{m}^{2}$; obese as BMI 30-34.99 $\mathrm{kg} / \mathrm{m}^{2}$, and severely obese $\left(>35 \mathrm{~kg} / \mathrm{m}^{2}\right)$. SPSS for Windows (version 22.0; SPSS, Inc., Chicago, IL, USA) was used to perform the statistical analyses.

\section{Results}

The characteristics of the study population are presented in Table 1 . The study population included primarily Hispanic BCS (63.6\%), had an average age of $52.7( \pm 10.4)$ years, and average BMI of $29.2 \pm 5.6 \mathrm{~kg} / \mathrm{m}^{2}$. The elevated BMI was due to the inclusion criteria of BMI 
$>25 \mathrm{~kg} / \mathrm{m}^{2}$ and/or body fat $>30 \%$ ), and may not precisely represent the whole BCS population. Overall, participants had an elevated BMI above normal $\left(18.5-24.9 \mathrm{~kg} / \mathrm{m}^{2}\right)$, and waist circumference $(96.9 \pm 13.1 \mathrm{~cm})$ greater than normal $(88 \mathrm{~cm})$. Seventeen participants had a normal BMI (18-24.99 kg/m²; 31\%), 21 participants were overweight BMI (25.0-29.9 $\left.\mathrm{kg} / \mathrm{m}^{2} ; 24 \%\right), 23$ participants were obese BMI $\left(30-34.99 \mathrm{~kg} / \mathrm{m}^{2} ; 26 \%\right)$ and 17 participants were severely obese BMI $\left(35 \mathrm{~kg} / \mathrm{m}^{2} ; 19 \%\right)$ The participants were diagnosed with mainly stage IA $(n=33: 37 \%)$ breast cancer, were postmenopausal $(n=50: 65 \%)$ and underwent a mastectomy $(\mathrm{n}=60: 78 \%)$.

We examined the differences in body composition by BIA and DXA in all participants (Table 2 and Figure 1). BIA and DXA provided significantly different body composition estimations. In particular, DXA provided significantly higher estimates of body fat percentage $(44.2 \pm 6.2$ vs $40.4 \pm 7.8 \%)$ and fat mass $(32.4 \pm 10.8$ vs $30.6 \pm 11.0 \mathrm{~kg})$ as well as a lower estimate of lean body mass $(39.1 \pm 7.6 \mathrm{vs} 42.9 \pm 5.9 \mathrm{~kg})$ compared to BIA $(\mathrm{P}<0.01)$. Body fat percentage measured by DXA was $8.6 \%$ higher compared to the estimates from BIA ( $\mathrm{P}<0.01)$. Fat mass measured by DXA was $9.7 \%$ higher compared to the estimates from BIA $(\mathrm{P}<0.01)$. In contrast, lean body mass measured by DXA was $5.5 \%$ lower compared to the value measured by BIA $(\mathrm{P}<0.01)$.

In order to assess differences among the two devices based on BMI, as noted in a previous study [14], participants were stratified by BMI categories: normal, overweight, obese and severely obese. Body composition values for DXA and BIA in the category of BMI are presented in Table 3. In BCS with normal BMI (Figure 2), DXA reported significantly higher estimates of body fat percentage $(38.1 \pm 4.3$ vs $32.0 \pm 5.4 \%)$ and fat mass $(21.7 \pm 5.6$ vs $19.1 \pm 4.5 \mathrm{~kg})$ compared to BIA $(\mathrm{P}<0.001)$ while DXA reported significantly lower lean body mass $(34.1 \pm 9.6$ vs $42.2 \pm 7.6 \mathrm{~kg})$ relative to $\mathrm{BIA}(\mathrm{P}<0.001)$.

The overweight BMI and obese BMI groups (Table 3) showed similar trends as the normal BMI group. In overweight BMI group (Figure 3), DXA reported significantly higher estimates of body fat percentage $(43.3 \pm 3.8$ vs $38.8 \pm 3.0 \%)$ and fat mass $(29.6 \pm 3.5 \mathrm{vs}$ $27.3 \pm 2.8 \mathrm{~kg}$ ) compared to BIA ( $\mathrm{P}<0.001)$ while DXA reported significantly lower lean body mass $(39.0 \pm 4.7$ vs $43.2 \pm 4.6 \mathrm{~kg}$ ) relative to BIA $(\mathrm{P}<0.001)$. In obese BMI group (Figure 4), DXA showed significantly $(\mathrm{P}<0.001)$ higher estimates of body fat percentage $(49.0 \pm 4.2$ vs $47.2 \pm 3.8 \%)$ and fat mass $(41.4 \pm 8.7$ vs $40.3 \pm 7.7 \mathrm{~kg})$ compared to BIA while DXA reported significantly $(\mathrm{P}<0.05)$ lower lean body mass relative to BIA $(42.6 \pm 5.4$ vs $44.8 \pm 6.6 \mathrm{~kg})$.

When examining the differences in the assessment of body composition in the severely obese group (Figure 5), body fat percentage and lean body mass were significantly $(\mathrm{P}<0.01)$ different between the two devices, with DXA reporting a higher body fat percentage (51.1 \pm 3.7 vs $49.6 \pm 2.8 \%)$ and lower lean body mass $(46.4 \pm 4.9$ vs $48.9 \pm 6.3 \mathrm{~kg})$ when compared to BIA $(\mathrm{P}<0.01)$. However, fat mass $(48.7 \pm 7.2 \mathrm{vs} 47.9 \pm 5.7 \mathrm{~kg})$ was not significantly different between the 2 devices $(\mathrm{P}>0.05)$. 


\section{Discussion}

Based on the search on PubMed, Web of Science, and Cochrane, this is the only study to date, which determined agreement between DXA and BIA in cancer survivors. We found that DXA and BIA provided significantly different estimations of body composition in BCS for fat mass, body fat percentage, and lean body mass. In particular, DXA showed higher estimations of body fat percentage and fat mass and lower estimations of lean body mass when compared to BIA. This implies that BIA may underestimate fat mass and body fat percentage without consideration of BMI in a population of BCS. Further, BIA provided significantly higher lean body mass than DXA, suggesting that BIA may overestimate lean body mass without consideration of BMI.

Using the Bland-Altman method, the present study indicated that there is a significant difference between BIA and DXA for determining body composition in overall BCS, except those within the severely obese BMI range. In severely obese BCS, DXA and BIA produced similar estimation of fat mass, which indicates that BIA may be preferred to BMI at the extremes of body composition. Our result is consistent with a previous study conducted by Volgyi et al., which examined the determination of fat mass and body fat percentage using DXA and two different BIA devices (InBody 720 and Tanita BC 418 MA) in men and women without serious metabolic, cardiovascular, or endocrine diseases.[14] The authors revealed that the DXA and BIA provided similar estimation of fat mass in their obese BMI group. However, body fat percentage and lean body mass were significantly different in our study. This may suggest that body composition measured via BIA varies depending on BMI categories, which is possibly due to the use of algorithms by BIA when determining fat mass and body fat percentage.

A high number of Hispanic (64\%) and postmenopausal women (58\%) were involved in the present study, A previous study has shown that menopausal status impacts the distribution of fat (higher visceral fat), rather than total fat mass and percentage,[17] suggesting that total fat mass and percentage may not be influenced by menopausal status. This may represent that our findings are applicable to overall BCS regardless of menopausal status. In our post hoc analysis, body fat percentage, lean body, and fat mass were not significantly different between premenopausal and postmenopausal BCS, regardless of BMI category (data not shown). This implies that our results may be applicable to overall BCS, regardless of menopausal status.

The small sample size may have affected reliability and bias outcomes to detect significant differences, particularly with regards to the BMI categories, thus findings in relation to these measures should be interpreted with some caution. Further studies are therefore required to consolidate these findings. Another limitation of this study is that we did not specifically control the hydration level although we instructed the participants to be fasting prior to body composition measures. This may result in misreporting and inaccuracy of data.

While BIA is an easy, non-invasive measure with low cost in clinical research settings, the use of BIA in clinical oncology settings can be limited due to the variation of protocols, different ethnic groups, body shape abnormalities, and possibly cancer treatment. In relation 
to our data, we included BCS who BMI $>25 \mathrm{~kg} / \mathrm{m}^{2}$ and $/$ or body fat $>30 \%$, which may have affected the ability to detect differences between groups. Thus, future studies utilizing the full spectrum of body habitus would be beneficial, and use of BIA requires further validation in clinical oncology conditions with specific consideration of BMI, ethnicity, cancer treatment and standardized protocol.

In summary, the body fat percentage, fat mass, and lean body mass of BCS differed when measured by BIA and DXA. Compared to DXA, BIA underestimated fat mass and overestimated lean body mass, suggesting that caution should be used when selecting which device to assess body composition in this population. In contrast, fat mass was similar between BIA and DXA in severely obese BCS. This implies that BIA needs to be used with caution to assess fat mass when BCS are recognized as severely obese by BMI. Future investigates are warranted to assess the utilization of these two devices in a larger cohort of BCS across BMI categories.

\section{Acknowledgments}

Funding: K07CA160718 from the National Cancer Institute, UL1TR001855 and UL1TR000130 from the National Center for Advancing Translational Science (NCATS) of the U.S. National Institutes of Health.

\section{References}

1. Frenzel AP, Pastore CA, Gonzalez MC: The influence of body composition on quality of life of patients with breast cancer. Nutr Hosp 2013, 28(5):1475-1482. [PubMed: 24160203]

2. Dieli-Conwright CM, Parmentier JH, Sami N, Lee K, Spicer D, Mack WJ, Sattler F, Mittelman SD: Adipose tissue inflammation in breast cancer survivors: effects of a 16-week combined aerobic and resistance exercise training intervention. Breast cancer research and treatment 2017.

3. Siegel RL, Miller KD, Jemal A: Cancer statistics, 2015 CA: a cancer journal for clinicians 2015, 65(1):5-29.

4. Czerniec SA, Ward LC, Meerkin JD, Kilbreath SL: Assessment of Segmental Arm Soft Tissue Composition in Breast Cancer-Related Lymphedema: A Pilot Study Using Dual Energy X-ray Absorptiometry and Bioimpedance Spectroscopy. Lymphat Res Biol 2015, 13(1):33-39. [PubMed: 25668060]

5. Deluche E, Leobon S, Desport JC, Venat-Bouvet L, Usseglio J, Tubiana-Mathieu N: Impact of body composition on outcome in patients with early breast cancer. Supportive care in cancer : official journal of the Multinational Association of Supportive Care in Cancer 2017.

6. Barrio AV, Eaton A, Frazier TG: A Prospective Validation Study of Bioimpedance with Volume Displacement in Early-Stage Breast Cancer Patients at Risk for Lymphedema. Annals of surgical oncology 2015, 22:S370-S375. [PubMed: 26085222]

7. Roberts CC, Levick JR, Stanton AW, Mortimer PS: Assessment of truncal edema following breast cancer treatment using modified Harpenden skinfold calipers. Lymphology 1995, 28(2):78-88. [PubMed: 7564495]

8. Riedl CC, Luft N, Bernhart C, Weber M, Bernathova M, Tea MKM, Rudas M, Singer CF, Helbich TH: Triple-Modality Screening Trial for Familial Breast Cancer Underlines the Importance of Magnetic Resonance Imaging and Questions the Role of Mammography and Ultrasound Regardless of Patient Mutation Status, Age, and Breast Density. Journal of Clinical Oncology 2015, 33(10): 1128-1134. [PubMed: 25713430]

9. Trevino-Aguirre E, Lopez-Teros T, Gutierrez-Robledo L, Vandewoude M, Perez-Zepeda M: Availability and use of dual energy X-ray absorptiometry (DXA) and bio-impedance analysis (BIA) for the evaluation of sarcopenia by Belgian and Latin American geriatricians. J Cachexia Sarcopeni 2014, 5(1):79-81. 
10. Popovic V, Zerahn B, Heaf J: Validity of Dual Energy X-Ray Absorptiometry and Bioimpedance in Assessing Body Composition and Nutrition in Peritoneal Dialysis Patients. Nephrol Dial Transpl 2015, 30 .

11. Glickman SG, Marn CS, Supiano MA, Dengel DR: Validity and reliability of dual-energy X-ray absorptiometry for the assessment of abdominal adiposity. Journal of applied physiology 2004, 97(2):509-514. [PubMed: 15075304]

12. Wells JCK, Williams JE, Fewtrell M, Singhal A, Lucas A, Cole TJ: A simplified approach to analysing bio-electrical impedance data in epidemiological surveys. Int J Obesity 2007, 31(3):507514.

13. Akyol M, Demir L, Alacacioglu A, Ellidiokuz H, Kucukzeybekl Y, Yildiz Y, Bayoglu V, Yildiz I, Salman T, Varol U et al.: The effects of adjuvant endocrine treatment on the serum leptin, adiponectin and body composition in patients with breast cancer [Izmir Oncology Group(IZOG) Study]. Eur J Cancer 2015, 51:S317-S317.

14. Volgyi E, Tylavsky FA, Lyytikainen A, Suominen H, Alen M, Cheng SL: Assessing body composition with DXA and bioimpedance: Effects of obesity, physical activity, and age. Obesity 2008, 16(3):700-705. [PubMed: 18239555]

15. Dieli-Conwright CM, Mortimer JE, Schroeder ET, Courneya K, Demark-Wahnefried W, Buchanan TA, Tripathy D, Bernstein L: Randomized controlled trial to evaluate the effects of combined progressive exercise on metabolic syndrome in breast cancer survivors: rationale, design, and methods. BMC cancer 2014, 14. [PubMed: 24410891]

16. Healthcare G: InBoby 520 Body Composition Analyzer 520 Instruction Manual [Online]. http:// www3gehealthcarecomsg/en-gb/products/categories/metabolic_health/ dsm_bioelectrical_impedance_analysis/inbody_520 Accessed July 152016.

17. Kanaley JA, Sames C, Swisher L, Swick AG, Ploutz-Snyder LL, Steppan CM, Sagendorf KS, Feiglin D, Jaynes EB, Meyer RA et al.: Abdominal fat distribution in pre- and postmenopausal women: The impact of physical activity, age, and menopausal status. Metabolism-Clinical and Experimental 2001, 50(8):976-982. [PubMed: 11474488] 
A

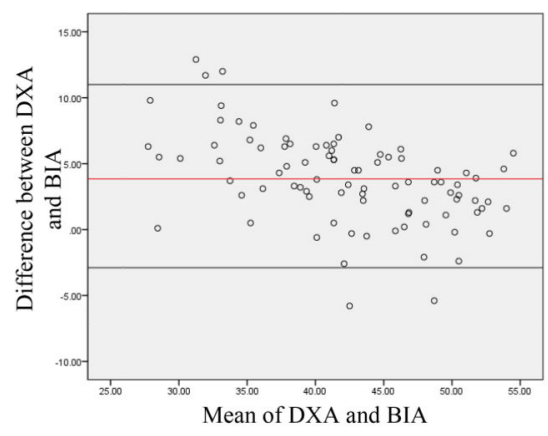

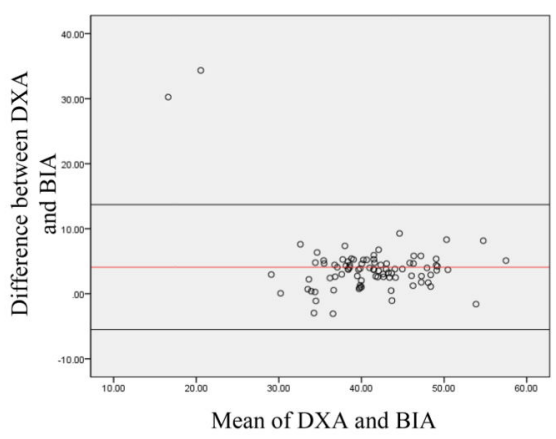

C

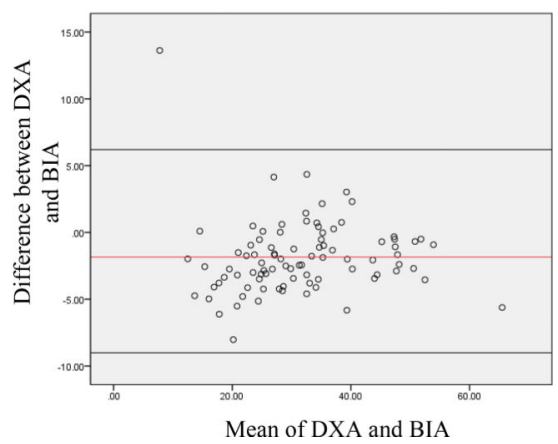

Figure 1.

Bland-Altman analysis plotted from body fat percentage (A) lean body mass (B) and fat mass (C) among all participants Abbreviations: BIA, Bio-electrical Impedance Analysis; DXA, Dual-Energy X-ray Absorptiometry 
A

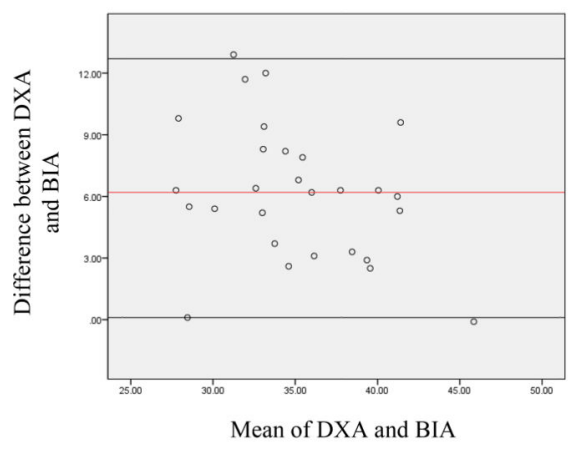

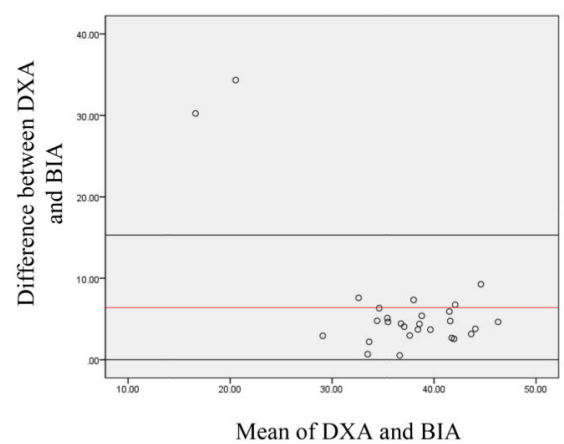

C

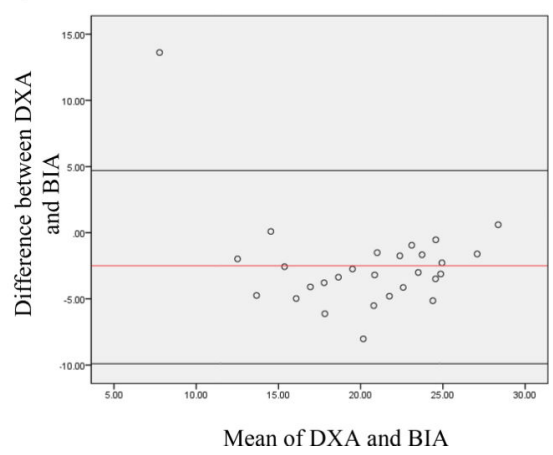

Figure 2.

Bland-Altman analysis plotted from body fat percentage (A) lean body mass (B) and fat mass (C) among BCS with normal BMI Abbreviations: BIA, Bio-electrical Impedance Analysis; DXA, Dual-Energy X-ray Absorptiometry 
A

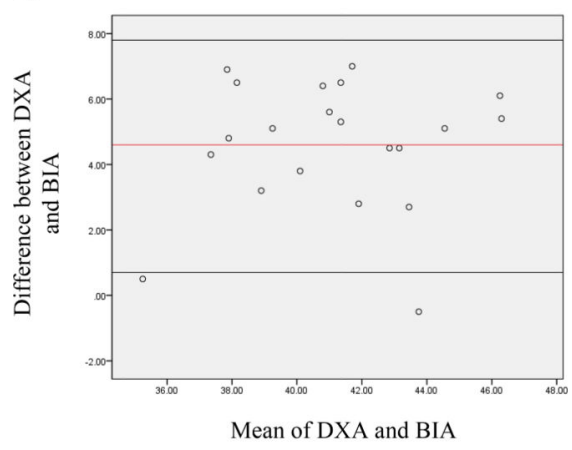

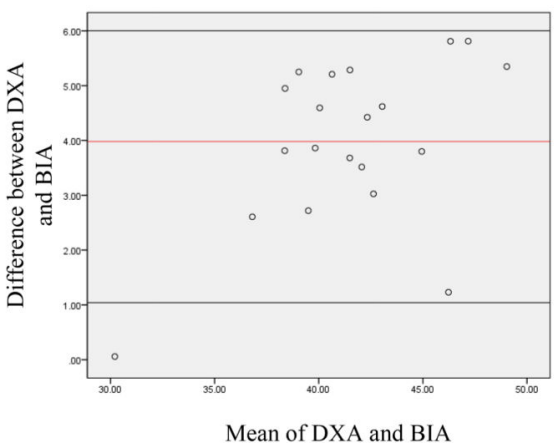

C

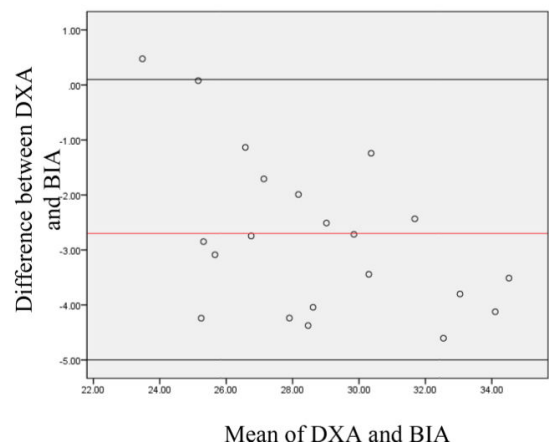

Figure 3.

Bland-Altman analysis plotted from body fat percentage (A) lean body mass (B) and fat mass (C) among overweight BCS Abbreviations: BIA, Bio-electrical Impedance Analysis; DXA, Dual-Energy X-ray Absorptiometry 
A

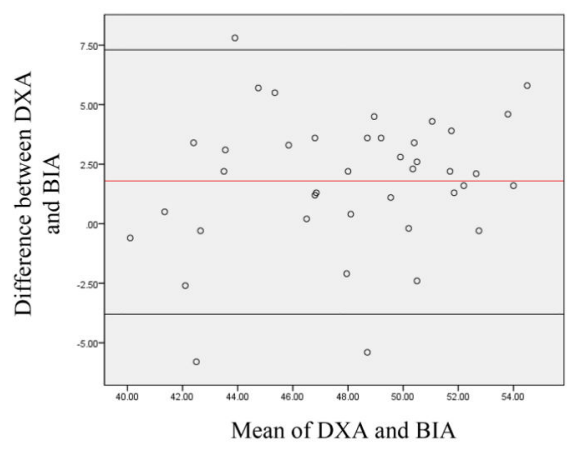

B

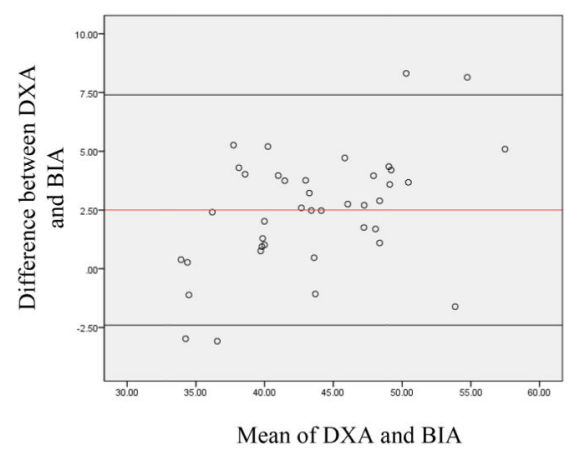

C

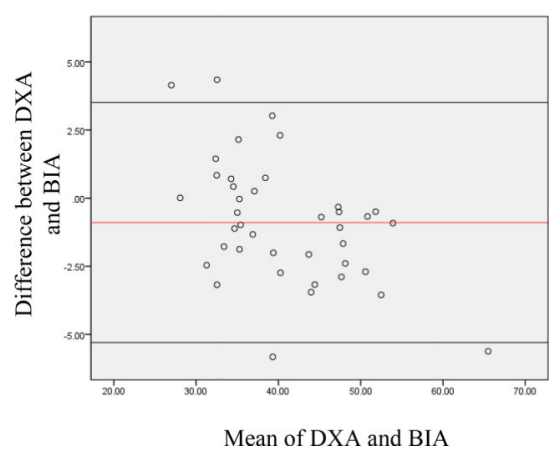

Figure 4.

Bland-Altman analysis plotted from body fat percentage (A) lean body mass (B) and fat mass (C) among obese BCS Abbreviations: BIA, Bio-electrical Impedance Analysis; DXA, Dual-Energy X-ray Absorptiometry 
A

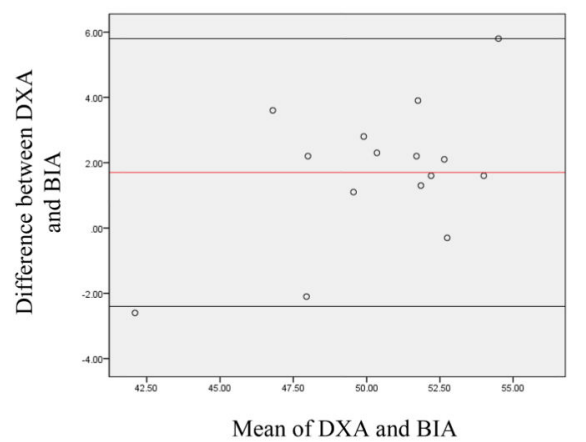

B

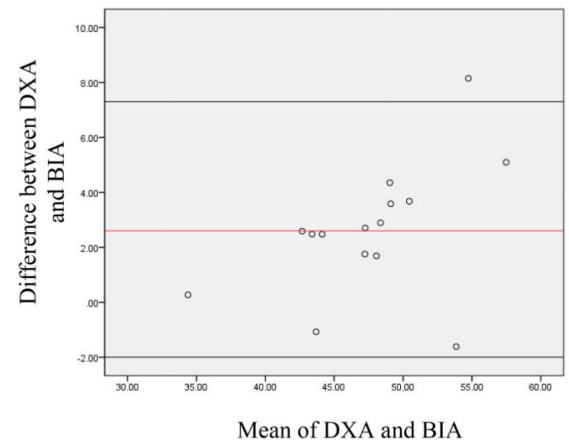

C

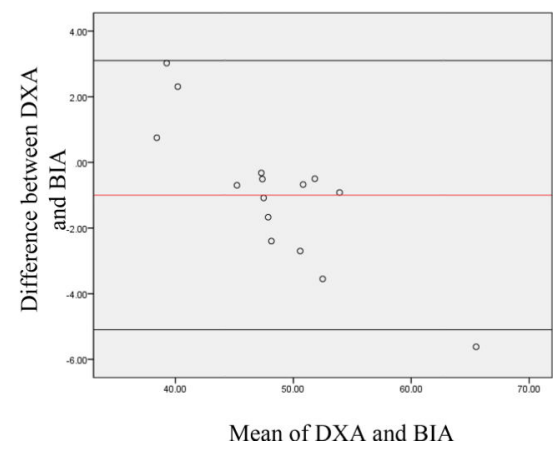

Figure 5.

Bland-Altman analysis plotted from body fat percentage (A) lean body mass (B) and fat mass (C) among severely obese BCS Abbreviations: BIA, Bio-electrical Impedance Analysis; DXA, Dual-Energy X-ray Absorptiometry 
Table 1.

Descriptive participant characteristics $(n=89)$.

\begin{tabular}{|c|c|}
\hline Age (years) ${ }^{*}$ & $52.7 \pm 10.4(29-76 y r)$ \\
\hline \multicolumn{2}{|l|}{ Race and Ethnicity, n (\%) } \\
\hline American Indian or Native & $0(0)$ \\
\hline Asian & $14(16)$ \\
\hline Black or African American & $3(3)$ \\
\hline Hispanic or Latino & $51(64)$ \\
\hline White & $21(19)$ \\
\hline BMI $\left(\mathrm{kg} / \mathrm{m}^{2}\right)^{*}$ & $29.2 \pm 5.6$ \\
\hline Normal (18.5-24.9) & $28(31)$ \\
\hline Overweight (25.0-29.9) & $21(24)$ \\
\hline Obese (30.0-35.0) & $23(26)$ \\
\hline Severely obese (>35.0) & $17(19)$ \\
\hline Body Weight (kg)* & $74.3 \pm 16.7$ \\
\hline Waist circumference $(\mathrm{cm})^{*}$ & $96.9 \pm 13.1$ \\
\hline Hip circumference $(\mathbf{c m}) *$ & $109.9 \pm 12.1$ \\
\hline \multicolumn{2}{|l|}{ Cancer stage, n (\%) } \\
\hline Stage I & $39(44)$ \\
\hline Stage II & $36(40)$ \\
\hline Stage III & $14(16)$ \\
\hline \multicolumn{2}{|l|}{ Prior chemotherapy, n (\%) } \\
\hline Yes & $54(61)$ \\
\hline No & $35(39)$ \\
\hline \multicolumn{2}{|l|}{ Surgery type, n (\%) } \\
\hline Mastectomy & $66(74)$ \\
\hline Lumpectomy & $23(26)$ \\
\hline \multicolumn{2}{|l|}{ Menopausal status, n (\%) } \\
\hline Premenopausal & $37(42)$ \\
\hline Postmenopausal & $52(58)$ \\
\hline
\end{tabular}

* Data presented as mean \pm SD. Abbreviation: BMI, body mass index 
Table 2.

Body composition by DXA and BIA in BCS.

\begin{tabular}{|c|c|c|c|}
\hline & Body fat $(\%)$ & Lean body mass $(\mathbf{k g})$ & Fat mass $(\mathbf{k g})$ \\
\hline DXA & $44.2 \pm 6.2$ & $39.1 \pm 7.6$ & $32.4 \pm 10.8$ \\
\hline BIA & $40.4 \pm 7.8$ & $42.9 \pm 5.9$ & $30.6 \pm 11.0$ \\
\hline P-value & \multicolumn{3}{|c|}{ P $<0.01^{*}$} \\
\hline
\end{tabular}

Abbreviations: BIA, Bio-electrical Impedance Analysis; DXA, Dual-Energy X-ray Absorptiometry

P value for Bland-Altman comparing multi frequency BIA to whole body DXA in 89 breast cancer survivors. The significance of P value shows that body compositions measured by two devices are significantly different. 
Table 3.

Body composition by DXA and BIA in BCS based on BMI categories

\begin{tabular}{|c|c|c|c|c|c|c|c|c|c|}
\hline \multirow{3}{*}{$\frac{\text { BMI }}{\text { Normal }}$} & \multicolumn{3}{|c|}{ Body fat (\%) } & \multicolumn{3}{|c|}{ Lean body mass (kg) } & \multicolumn{3}{|c|}{ Fat mass (kg) } \\
\hline & DXA & $38.1 \pm 4.3$ & $\mathrm{P}<0.001^{*}$ & DXA & $34.1 \pm 9.6$ & $\mathrm{P}<0.001^{*}$ & DXA & $21.7 \pm 5.6$ & $\mathrm{P}<0.001$ * \\
\hline & BIA & $32.0 \pm 5.4$ & & BIA & $42.2 \pm 7.6$ & & BIA & $19.1 \pm 4.5$ & \\
\hline \multirow[t]{2}{*}{ Overweight } & DXA & $43.3 \pm 3.8$ & \multirow[t]{2}{*}{$\mathrm{P}<0.001^{*}$} & DXA & $39.0 \pm 4.7$ & \multirow[t]{2}{*}{$\mathrm{P}<0.001 *$} & DXA & $29.6 \pm 3.5$ & \multirow[t]{2}{*}{$\mathrm{P}<0.001$ * } \\
\hline & BIA & $38.8 \pm 3.0$ & & BIA & $43.2 \pm 4.6$ & & BIA & $27.3 \pm 2.8$ & \\
\hline \multirow[t]{2}{*}{ Obese } & DXA & $49.0 \pm 4.2$ & \multirow{2}{*}{$\mathrm{P}<0.001^{*}$} & DXA & $42.6 \pm 5.4$ & \multirow{2}{*}{$\mathrm{P}<0.001^{*}$} & DXA & $41.4 \pm 8.7$ & \multirow[t]{2}{*}{$\mathrm{P}<0.05^{*}$} \\
\hline & BIA & $47.2 \pm 3.8$ & & BIA & $44.8 \pm 6.6$ & & BIA & $40.3 \pm 7.7$ & \\
\hline \multirow[t]{2}{*}{ Severely Obese } & DXA & $51.1 \pm 3.7$ & \multirow[t]{2}{*}{$\mathrm{P}<0.01^{*}$} & DXA & $46.4 \pm 4.9$ & \multirow[t]{2}{*}{$\mathrm{P}<0.01^{*}$} & DXA & $48.7 \pm 7.2$ & \multirow[t]{2}{*}{$\mathrm{P}=0.102$} \\
\hline & BIA & $49.6 \pm 2.8$ & & BIA & $48.9 \pm 6.3$ & & BIA & $47.9 \pm 5.7$ & \\
\hline
\end{tabular}

Abbreviations: BIA, Bio-electrical Impedance Analysis; BMI, body mass index; DXA, Dual-Energy X-ray Absorptiometry;

* $\mathrm{P}$ value for Bland-Altman comparing multi frequency BIA to whole body DXA in 89 breast cancer survivors. The significance of P value shows that body compositions measured by two devices are significantly different.

${ }^{\dagger}$ Total body water: Normal BMI: $29.5 \pm 3.6 \mathrm{~kg}$, overweight BMI: $31.7 \pm 3.2 \mathrm{~kg}$, obese BMI: $31.2 \pm 3.8 \mathrm{~kg}$, and severely obese BMI: $36.6 \pm 4.5 \mathrm{~kg}$ 